\title{
New Definition of Sepsis
}

Xiaofeng Shen, * Fuzhou Wang *

SEPSIS OR SEPTIC shock is one of the major causes of mortality in intensive care medicine. How to define and how to make an accurate diagnosis possess critical implications for patients and intensive caregivers. With the development of modern medical science, new challenges rose for how to re-define sepsis and also revision is needed. Should we add organ dysfunction to the diagnostic criteria of systemic inflammatory response syndrome, and whether are there early symptoms or signs of organ dysfunction need to be considered critically. May be the new definition of sepsis can save more lives.
*Department of Anesthesiology and Critical Care Medicine, Nanjing Medical University, Nanjing 210004, China

$\Delta$ Correspondence to Fuzhou Wang, No. 123, Tianfei Xiang, Mochou Rd., Nanjing 210004, China

Tel: +86-25-5222 6112

Fax: $+86-25-84200723$

Email: zfwang50@njmu.edu.cn

SCIENCE INSIGHTS 2013; 2(1): 37.

Keywords: Sepsis - Septic shock - Intensive medicine - Definition

\section{To the Editor,}

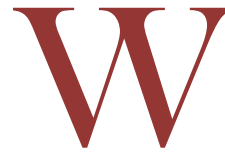
E strongly agree the perspective from Drs Vincent et al. on the definition of sepsis as "a systemic response to infection with the presence of some degree of organ dysfunction" that was published on March 022013 in The Lancet (1). We believe such an addition of some degree of organ dysfunction to the conventional definition will make it more precise in defining a patient whether developed sepsis or not merely on the criteria of sys-

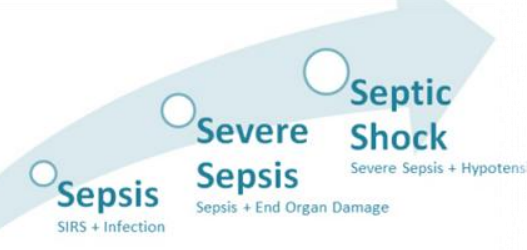

SIRS

Temp. $>38^{\circ} \mathrm{C}$ or $<36^{\circ} \mathrm{C}, \mathrm{HR}>90, \mathrm{RR}>20$ or $\mathrm{PaCO}_{2}<32$,

temic inflammatory response syn- drome (SIRS) bases. The beneficial effect for such a change would be on 1) patients' outcomes because we would become alert to patient's real situation but not only the SIRS if organ dysfunction was added to the diagnostic criteria, which will subsequently make critical care physicians considered more carefully when treating these patients because they had some degree of organ dysfunction, 2) clinical trials' reliability, the enrolling criteria based on SIRS definition is pretty sensitive if without such a change that would result in many patients were recruited without de-facto sepsepsis. Beside these good aspects, we still consider that what degree of the organ dysfunction should be defined as pointed out by Drs Vincent et al that "some degree of organ dysfunction", i.e. how to define the "some degree". In fact, it is a continual sequential process from
SIRS to sepsis to severe sepsis to septic shock to multiple organ dysfunction syndromes (2). Therefore, it is difficult to give a precise definition of the "some degree's organ dysfunction" for each of the period of the whole disease development.

\section{Conflict of Interests}

None

\section{References}

1. Vincent JL, Opal SM, Marshall JC, Tracey KJ. Sepsis definitions: time for change. Lancet 2013; 381: 7745.

2. Fry DE. Sepsis, systemic inflammatory response, and multiple organ dysfunction: the mystery continues. Am Surg 2012; 78: 1-8.

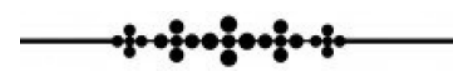

